\title{
The simple failure of Curie's principle
}

\author{
Bryan W. Roberts \\ usc.edu/bryanroberts \\ Forthcoming in Philosophy of Science
}

July 3, 2013

\begin{abstract}
I point out a simple sense in which the standard formulation of Curie's principle is false, when the symmetry transformation it describes is time reversal.
\end{abstract}

\section{INTRODUCTION}

John Earman has suggested that there is a simple formulation of Curie's principle that is not only deeply intuitive but "virtually analytic" (Earman 2004, p.173). He is not the only one to take this view?, but gives one of its clearest statements. Earman formulates Curie's principle as the claim: If,

(CP1) the laws of motion/field equations governing the system are deterministic;

(CP2) the laws of motion/field equations governing the system are invariant under a symmetry transformation; and

Acknowledgements. For their helpful comments and suggestions I would like to thank John Earman, John D. Norton, and the December 8, 2012 audience at the Southern California Philosophy of Physics Reading Group at U.C., Irvine.

${ }^{1}$ Curie himself took it to be an a priori truth that, "[w]hen certain effects show a certain asymmetry, this asymmetry must be found in the causes which gave rise to them" (Curie 1894), translation from (Brading and Castellani 2003, p.311313). The meaning of this statement is vague, because the words "cause" and "effect" here are not defined. But on the plausible reading that an "effect" is a state, and a "cause" is another state related to the first by a deterministic law, we immediately get the formulation presented by Earman. Similar readings of Curie can be found in Mittelstaedt and Weingartner (2005, p.231), where it is said that, "from an asymmetric effect and symmetric laws we may conclude asymmetric initial conditions," and Ismael (1997, p.170), who claims to have proven that "all characteristic symmetries of a Curie-cause are also characteristic symmetries of its effect." 
(CP3) the initial state of the system is invariant under said symmetry; then

(CP4) the final state of the system is also invariant under said symmetry. (Earman 2004, p.176)

Speaking intuitively, one might summarize the principle: if no asymmetry goes in, then no asymmetry comes out.

I would like to point out a simple sense in which this formulation of Curie's principle fails, when the language therein is interpreted in the standard way, and the symmetry transformation is time reversal. I will begin by illustrating a very simple counterexample in classical Hamiltonian mechanics, and then show how such counterexamples are endemic to quantum mechanics and quantum field theory. I discuss three alternative interpretations of Curie's principle that aim to resist the conclusion that the principle fails for time reversal, and argue that none are satisfying. I conclude that one must apply Curie's principle with care, as it only applies for a particular kind of symmetry transformation that does not include time reversal.

\section{FAILURE IN CLASSICAL MECHANICS}

2.1. In pictures. Take a harmonic oscillator, like a bob on a spring. It is manifestly time reversal invariant, in that for every possible motion of the bob, there is a "time-reversed motion" that is also possible.

How does the instantaneous state of an ordinary classical system like this one change under time reversal? The standard textbook answer is that the position of the state remains unchanged, while the direction of the momentum is reversed. One can get the intuition for this by imagining that we film the motion of the bob, and then play the film in reverse. To determine what time reversal does at an instant we look at a single "frame" of the film and ask how it changes when we view the reversed film. The answer is that, since rightward motion in the original film becomes leftward in the reversed film, the momenta simply reverse sign. We thus say that an instantaneous state of an ordinary classical system is "invariant" (or "unchanged" or "preserved") under time reversal if and only if the momentum of that state is zero.

Let us now suppose that this particular bob-spring system begins its motion at time $t=0$ with the spring compressed out of equilibrium, and with no initial momentum, as in Figure 1(a), The bob then springs back in the other direction, acquiring some non-zero momentum, as in Figure 1(b), How does time reversal transform these initial and final states? 


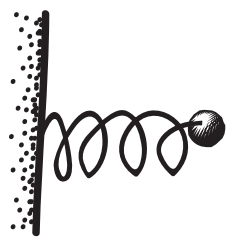

(a) Initial state

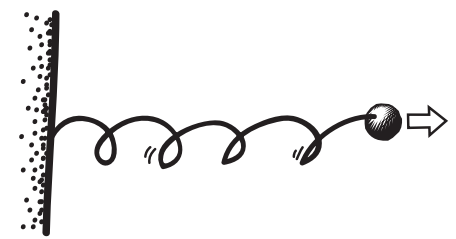

(b) Final state

Figure 1. (a) A harmonic oscillator initially compressed out of equilibrium with zero momentum. (b) A final state for which the system has non-zero momentum.

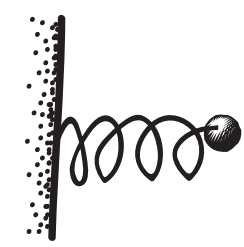

(a) Time reversed initial state

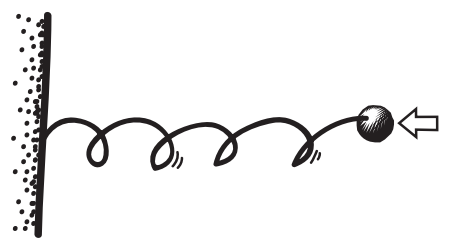

(b) Time reversed final state

Figure 2. (a) The initial state has no momentum, and so is preserved by time reversal. (b) A final state has non-zero momentum, and so is not preserved by time reversal.

Our initial state has zero momentum, so it is preserved by the time reversal operator, as in Figure 2(a). But the final state has non-zero momentum, which reverses direction under the time reversal operator, as in Figure 2(b). The result: the laws of motion for the harmonic oscillator are time reversal invariant, and the initial state is preserved by the time reversal operator, but the final state is not. Curie's principle fails when the symmetry is time reversal, in systems as simple as the harmonic oscillator.

2.2. Mathematical verification. Let's do the exercise of checking this result in Hamiltonian mechanics. The possible states of the harmonic oscillator are the possible values for the position and momentum $(q, p)$ of the bob in phase space. The laws of motion for the system are Hamilton's equations,

$$
\frac{d}{d t} q(t)=\frac{\partial}{\partial p} h(q, p), \quad \frac{d}{d t} p(t)=-\frac{\partial}{\partial q} h(q, p) .
$$


The Hamiltonian $h(q, p)$ for the harmonic oscillator is $h(q, p)=(1 / 2 m) q^{2}+$ $(k / 2) p^{2}$, where $m$ and $k$ are constants. For simplicity, consider an oscillator for which $m=1 / 2$ and $k=2$, so that $h(q, p)=q^{2}+p^{2}$. The laws of motion for this system are manifestly time reversal invariant, in that if $(q(t), p(t))$ is a possible trajectory, then $(q(-t),-p(-t))$ is a possible trajectory as well ${ }^{2}$.

We now need to check that there is a trajectory with an initial state that is preserved by time reversal, and a final state that is not. One such trajectory is the following, which one can check $\mathrm{S}^{3}$ is a solution to the laws of motion above:

$$
q(t)=\cos (2 t), \quad p(t)=-\sin (2 t) .
$$

At time $t=0$, this system has zero momentum, since $p(0)=\sin (0)=0$. But it has non-zero momentum for the subsequent times $0<t<2 \pi$. The time reversal operator $T:(q, p) \mapsto(q,-p)$ therefore preserves the initial state, but not all later states.

2.3. Summary. Here is what we have observed in the example above:

(1) The harmonic oscillator is time reversal invariant. This is a simple mathematical fact about the law of motion for the harmonic oscillator.

(2) We chose a trajectory with an initial state that is preserved by time reversal. In particular, we chose a trajectory for which the harmonic oscillator is not always in equilibrium, and then picked an initial state with zero momentum.

(3) Not all later states of that trajectory are so preserved. The later states of the harmonic oscillator have non-zero momentum, and so are not preserved by the time reversal operator.

Curie's principle thus fails when the symmetry transformation is time reversal. Let me now say briefly what the origin of this failure is in mathematical terms.

\footnotetext{
${ }^{2}$ To verify: Let $(q(t), p(t))$ be a solution to Hamilton's equations. The Hamiltonian $h(q, p)=p^{2}+q^{2}$ has the property that $h(q, p)=h(q,-p)$. So, Hamilton's equations also hold for $h(q,-p)$. But Hamilton's equations hold for all values of $t$, and therefore under the substitution $t \mapsto-t$. Making this substitution, we thus find that $-(d / d t) q(-t)=\partial h(q, p) / \partial p$ and hence that $(d / d t) q(-t)=\partial h(q,-p) / \partial(-p)$; similarly, $-(d / d t) p(-t)=-\partial h(q, p) / \partial q$, and hence $(d / d t)(-p(-t))=-\partial h(q,-p) / \partial q$. That is, $(q(-t),-p(-t))$ is also a solution to Hamilton's equations.

${ }^{3}$ Namely, $d q / d t=(d / d t)(\cos (2 t))=-2 \sin (2 t)=2 p(t)=\partial h / \partial p$, and $d p / d t=$ $(d / d t)(-\sin (2 t))=-2 \cos (2 t)=-2 q(t)=-\partial h / \partial q$.
} 
Hamiltonian mechanics comes equipped with an object called a symplectic form; it can be written $d q \wedge d p$ in Darboux coordinates. Symmetry transformations in Hamiltonian mechanics include not only symplectic transformations, which leave the symplectic form invariant, but also antisymplectic transformations, which reverse its sign. It is easy to see that the time reversal transformation $T(q, p)=(q,-p)$ is antisymplectic, because it reverses the sign of the symplectic form.

Mathematically, the problem for Curie's principle comes down to this. A system with a Hamiltonian $h$ might be invariant under a transformation $S(q, p) \mapsto\left(q^{\prime}, p^{\prime}\right)$ that is symplectic or antisymplectic. It would be standard parlance to call the transformation a "symmetry" either way. If $S$ is symplectic, then it follows that $S$-invariant states evolve to $S$-invariant states. But this conclusion does not follow when $S$ is antisymplectic. Time reversing the harmonic oscillator provides just one simple example of this.

\section{Failure in QuAntum TheOry}

Curie's principle fails just as badly in quantum theory. Before illustrating this, I'll describe the standard definition of time reversal and time reversal invariance in the quantum context, since they may be unfamiliar. Then, rather than redo the harmonic oscillator example above in the context of quantum theory, I'll illustrate a general class of systems (of which the harmonic oscillator is just one example) for which Curie's principle fails, and summarize the character of this failure just as I did for the classical case.

3.1. Time reversal in quantum theory. Curie's principle fails quite generally in both non-relativistic quantum mechanics and in relativistic quantum field theory. To keep the discussion general enough to apply to both, I will characterize the spacetime on which quantum theory takes place as an affine space $\mathcal{M}$, which admits a foliation into spacelike hypersurfaces. This will allow us to think of $\mathcal{M}$ as either a non-relativistic spacetime (such as Newtonian or Galilei spacetime), or a relativistic spacetime (such as Minkowski spacetime).

The vector states of a quantum system will be described by vectors in a Hilbert space $\mathcal{H}$. For any foliation $\Sigma_{t}$ of the spacetime $\mathcal{M}$ into spacelike hypersurfaces, we take there to be a continuous one-parameter group of unitary operators $\mathcal{U}_{t}=e^{-i t H}$, which describes how a state $\psi \in \mathcal{H}$ changes by the rule,

$$
\psi(t)=e^{-i t H} \psi
$$


In differential form, this law becomes the familiar Schrödinger equation $i(d / d t) \psi(t)=H \psi(t)$, which holds for all $\psi(t)$ in the domain of $H$.

What do time reversal and time reversal invariance mean in this context? Time reversal in quantum mechanics takes a trajectory $\psi(t)$ to a new trajectory $T \psi(-t)$, where $T: \mathcal{H} \rightarrow \mathcal{H}$ is a bijection called the time reversal operator. This operator $T$ has the special property of being antiunitary. Like a unitary operator, an antiunitary operator satisfies $T^{*} T=T T^{*}=I$. But unlike a unitary operator it is antilinear, meaning that for any two vectors $\psi$ and $\phi$ and for any complex constants $a$ and $b$,

$$
T(a \psi+b \phi)=a^{*} T \psi+b^{*} T \phi
$$

For completeness, let me briefly rehearse one simple argument for why time reversal has this unusual property. We will make use of a representation of the canonical commutation relations $[Q, P] \psi=i \psi$, although such a representation is not necessary, and considerably more general arguments can be given 4 . As before, assume that by reversing time "at an instant," we preserve positions while reversing momenta: $T Q T^{-1}=Q$ and $T P T^{-1}=-P$. Applying $T$ to both sides of the commutation relations then immediately implies that,

$$
T i T^{-1} \psi=T[Q, P] T^{-1} \psi=\left[T Q T^{-1}, T P T^{-1}\right] \psi=[Q,-P] \psi=-i \psi .
$$

No linear operator can reverse the sign of the complex constant $i$. Moreover, Wigner's theorem requires that all candidates for symmetry transformations be linear or antilinear. So, since $T$ cannot be linear, it must be antilinear.

What now does it mean to say that a quantum system is time reversal invariant? As in classical mechanics, we say that a quantum system $\left(\mathcal{H}, e^{-i t H}\right)$ is time reversal invariant if and only if, whenever $\psi(t)$ is a solution to the law of motion, then so is $T \psi(-t)$. This is equivalent ${ }^{5}$ to the statement,

$$
T H T^{-1}=H
$$

where $H$ is the generator (the "Hamiltonian") appearing in the unitary dynamics $\mathcal{U}_{t}=e^{-i t H}$.

\footnotetext{
${ }^{4}$ The original textbook treatment of Wigner $(1931, \S 20)$ remains one of the best. Excellent modern treatments have also been given by Sachs $(1987, \S 3.2)$ and Weinberg $(1995, \S 2.6)$.

${ }^{5}$ This was pointed out, for example, in (Earman 2002, p.248).
} 
3.2. Curie's principle in quantum theory. The quantum harmonic oscillator provides a counterexample to Curie's principle just like the classical harmonic oscillator does. Carrying the example over into the quantum context is a simple exercise. So, instead of doing this, let me illustrate a more general class of quantum system $\left(\mathcal{H}, e^{-i t H}\right)$ for which Curie's principle fails. This class will include the harmonic oscillator as a special case. Namely, consider any quantum system that satisfies:

(i) time reversal invariance, $T H T^{-1}=H$, and

(ii) non-degeneracy, that $H \psi_{1}=h \psi_{1}$ and $H \psi_{2}=h \psi_{2}$ only if $\psi_{1}=$ $e^{i \theta} \psi_{2}$ for some $\theta \in[0,2 \pi]$.

Non-degeneracy says that if two states have the same value of energy, then they must be related by a phase-factor $e^{i \theta}$, and thus actually represent the same probabilistic stat ${ }^{6}$. A Hamiltonian is non-degenerate if two distinct energy states never occupy the same energy level.

We will show that for the time reversal symmetry transformation, any system satisfying (i) and (ii) provides a counterexample to Curie's principle. We first construct an initial state $\phi$ that is preserved by $T$, and then exhibit a final state $\phi(t)$ that is not preserved by $T$. The first step will make use of the following fact about such systems: these systems generally admit at least two distinct states $\phi_{1}, \phi_{2} \in \mathcal{H}$ such that $T \phi_{1}=\phi_{1}$ and $T \phi_{2}=\phi_{2}$. This fact can be derived by noting that if $\psi_{1}$ is an energy eigenvector $\left(H \psi_{1}=h_{1} \psi_{1}\right)$, then by time reversal invariance,

$$
H\left(T \psi_{1}\right)=T H \psi_{1}=T h_{1} \psi_{1}=h_{1}\left(T \psi_{1}\right) .
$$

That is, $T \psi_{1}$ is also an eigenstate of $H$ with eigenvalue $h_{1}$. But since $H$ is non-degenerate, this implies that $T \psi_{1}=e^{i \theta} \psi_{1}$ for some phase factor $e^{i \theta}$. Let $\phi_{1}:=e^{i \theta / 2} \psi_{1}$. Since $T$ is antilinear, $T e^{i \theta / 2}=e^{-i \theta / 2} T$. The energy eigenstate $\phi_{1}$ thus has the property that,

$$
T \phi_{1}=T e^{i \theta / 2} \psi_{1}=e^{-i \theta / 2} T \psi_{1}=e^{-i \theta / 2} e^{i \theta} \psi_{1}=e^{i \theta / 2} \psi_{1}=\phi_{1} .
$$

By repeating this procedure with an energy eigenvector $\psi_{2}$ that is orthogonal to $\psi_{1}$, we get a second state $\phi_{2}$ such that $T \phi_{2}=\phi_{2}$ as well.

The violation of Curie's principle can now be seen by setting our initial state $\phi$ to be the superposition of the two orthogonal energy

\footnotetext{
${ }^{6}$ Vectors related by a phase factor occupy the same ray, defined by a set $\Psi:=$ $\left\{e^{i \theta} \psi: \theta \in[0,2 \pi]\right\}$. Vectors on the same ray have the same probabilistic content, in that the transition probability $|\langle\phi, \psi\rangle|^{2}$ remains the same when $\psi$ is replaced with another vector $e^{i \theta} \psi$ on the same ray (and similarly for $\phi$ ). It is for this reason we often say a pure state in ordinary quantum mechanics is best represented by a ray, rather than a vector.
} 
eigenvectors $\phi_{1}$ and $\phi_{2}$ :

$$
\phi=\frac{1}{\sqrt{2}}\left(\phi_{1}+\phi_{2}\right) .
$$

Since $T \phi_{1}=\phi_{1}$ and $T \phi_{2}=\phi_{2}$, it follows that $T \phi=\phi$ as well. So, our initial state $\phi$ is preserved by the time reversal operator. Indeed, this initial state is preserved by $T$ in a more general sense as well, in that the ray $\Phi=\left\{e^{i \theta} \phi: \theta \in[0,2 \pi]\right\}$ is left unchanged by the transformation that applies $T$ to each element.

We can now verify that the final state $\phi(t)$ is not generally preserved by $T$, contrary to the conclusion of Curie's principle. After a length of time $t$, the state $\phi$ evolves to

$$
\phi(t)=\frac{1}{\sqrt{2}}\left(e^{-i t h_{1}} \phi_{1}+e^{-i t h_{2}} \phi_{2}\right),
$$

where $h_{1}$ and $h_{2}$ are the energy eigenvalues of $\phi_{1}$ and $\phi_{2}$, respectively. Again applying the consequence of antilinearity that $T e^{-i t h}=e^{i t h} T$, we get that,

$$
T \phi(t)=\frac{1}{\sqrt{2}}\left(T e^{-i t h_{1}} \phi_{1}+T e^{-i t h_{2}} \phi_{2}\right)=\frac{1}{\sqrt{2}}\left(e^{i t h_{1}} \phi_{1}+e^{i t h_{2}} \phi_{2}\right) .
$$

This expression is not equal to $\phi(t)$ when $t \in(0,2 \pi)$. It does not even lie on the same ray. So, although $T$ preserves the initial state defined in Equation (3), it does not generally preserve the final state defined in Equation (4), in spite of the fact that the system is time reversal invariant.

Thus, any system that is both time reversal invariant and nondegenerate provides a counterexample to Curie's principle. As is wellknown 7 , the quantum harmonic oscillator satisfies both these conditions, and is therefore just a special case of this general failure.

3.3. Summary. By considering an arbitrary time reversal invariant system with a non-degenerate Hamiltonian, we have observed:

(1) Such systems are time reversal invariant, by explicit assumption.

(2) We chose a trajectory with an initial state that is preserved by time reversal. In particular, we constructed a superposition of energy eigenstates that is preserved by the time reversal operator $T$.

(3) Not all later states on that trajectory are so preserved. We showed that this superposition evolves to states that are not preserved by $T$.

${ }^{7}$ Cf. (Messiah 1999, §XII.4) 
Thus, Curie's principle fails for quantum mechanics as well. As in the classical case, the mathematical root of the problem lies in the special nature of time reversal in quantum theory. This time it is the antiunitary character of time reversal.

According to standard usage of the term, a transformation is a "symmetry" if it is unitary or if it is antiunitary. If a system is invariant under a symmetry that is unitary, then we can immediately conclude that $S$-symmetric states evolve to $S$-symmetric states. This is because, for unitary operators, $S H S^{-1}=H$ implies that $S e^{-i t H} S^{-1}=e^{-i t H}$, and hence that,

$$
S \psi=\psi \Rightarrow S \psi(t)=S\left(e^{-i t H} \psi\right)=e^{-i t H} S \psi=\psi(t) .
$$

In other words, Curie's principle is true for unitary operators! But the conclusion does not follow for antiunitary operators. In particular, if $T$ is antiunitary, then $T H T^{-1}=H$ implies that $T e^{-i t H} T^{-1}=e^{i t H}$, because conjugating the complex number reverses its sign. One can infer from $T H T^{-1}=H$ that,

$$
T \psi=\psi \Rightarrow T \psi(t)=T\left(e^{-i t H} \psi\right)=e^{i t H} T \psi=\psi(-t) .
$$

But it is not generally the case that $T \psi(t)=\psi(t)$, as Curie's principle would have it. Indeed, this conclusion would only be true in general if $\psi(t)=\psi(-t)$, which holds only of a trajectory $\psi(t)=\psi$ that remains unchanged for all tim $\AA^{8}$. So, if a time reversal invariant quantum system is interesting enough to allow a state that is preserved by time reversal to change over time, then that system is a counterexample to Curie's principle.

\section{Three ATtempts to Resist FAILURE}

The simple conclusion that I would like to defend is that Curie's principle is not generally true: the principle is formulated for an arbitrary symmetry, but it is false when that symmetry is time reversal. I would now like to discuss several true principles that are in the neighborhood of Curie's. Given their similarity to the formulation discussed so far, one might wonder if it is possible to resist the failure of Curie's principle through some clever interpretive maneuvering. I will argue here

\footnotetext{
${ }^{8}$ Proof: Let $\psi(t)$ (for $t \in \mathbb{R}$ ) be a trajectory. Suppose that for every choice of initial state $\phi$ on that trajectory, $\phi(t)=\phi(-t)$ for all $t$, where $\phi(t):=e^{-i t H} \phi$. For any fixed time $t$, define $\phi:=e^{-i(t / 2) H} \psi$. Then by assumption $\phi(t / 2)=\phi(-t / 2)$. But $\phi(t / 2)=e^{-i(t / 2) H} \phi=e^{-i(t / 2) H} e^{-i(t / 2) H} \psi=\psi(t)$, while $\phi(-t / 2)=e^{i t H} e^{-i t H} \psi=$ $\psi$. Therefore, $\psi(t)=\psi$.
} 
that it is not. There are at least three ways to reinterpret Curie's principle to get a true proposition. None appear to provide a satisfactory interpretation of the principle.

4.1. Argue time reversal is not a symmetry. One way to reinterpret Curie's principle is to modify what counts as a "symmetry transformation." The basic strategy is to say that, in the context of quantum theory, only unitary transformations can be symmetry transformations, and time reversal is not unitary. This allows one to avoid the counterexamples altogether, by expelling time reversal (and all antiunitary transformations) from the garden of symmetries.

The strategy can be applied in classical mechanics as well. Curie's principle fails for antisymplectic transformations in classical mechanics in just the same way that it fails for antiunitary transformations in quantum mechanics. So, by requiring that all symmetries be symplectic, we can make the world safe for Curie's principle in classical mechanics too.

Earman has formulated a statement of Curie's principle that might be seen as adopting this view in the algebraic framework for quantum field theory. He begins with an algebra of observables, together with an automorphism group $\alpha$ describing the dynamics. His approach is then to characterize a "symmetry transformation" in quantum field theory as a (linear) automorphism $\theta$ of the $C^{*}$ algebra. In this framework, Earman writes:

Proposition 2 (Curie's principle). Suppose that the initial state $\omega_{o}$ is $\theta$-symmetric (i.e. $\widehat{\theta \omega_{o}}:=\omega_{o} \circ \theta=\omega_{o}$ ) and that the dynamics $\alpha$ is also $\theta$-symmetric (i.e. $\theta \alpha \theta^{-1}=$ $\alpha)$. Then the evolved state $\omega_{1}:=\widehat{\alpha \omega_{o}}$ is $\theta$-symmetric. (Earman 2004, p.198)

This certainly resembles Curie's principle: the dynamics are deterministic (CP1), the dynamics are preserved by a symmetry (CP2), the initial state is preserved by the symmetry (CP3), and we conclude that the final state is preserved by the same symmetry (CP4). An easier special case of this can be formulated in non-relativistic quantum mechanics. There, the approach would be to characterize a symmetry transformation $\theta$ as a (linear) unitary transformation on a Hilbert space $\mathcal{H}$. Then we have:

Non-Relativistic Proposition 2. Suppose that the initial state $\psi_{0} \in \mathcal{H}$ is $\theta$-symmetric (i.e. $\theta \psi_{0}=\psi_{0}$ ) and that the unitary group $e^{-i t H}$ generating the dynamics is also 
$\theta$-symmetric (i.e. $\theta e^{-i t H} \theta^{-1}=e^{-i t H}$ ). Then the evolved state $\psi_{1}:=e^{-i t H} \psi_{0}$ is $\theta$-symmetric.

Both of these propositions are mathematically correct, and their proofs are trivial ${ }^{9}$. Time reversal is excluded from the content of both propositions because it is antilinear.

Although Earman's approach results in a Curie-like principle, it is at the expense of the orthodox definition of symmetry transformations. In quantum theory, symmetry transformations include not only the unitary transformations (which are linear), but also antiunitary transformations (which are antilinear). In the algebraic framework in which Earman works, symmetry transformations include both automorphisms and anti-automorphisms. This is the orthodox view of symmetries, arising out of Wigner's theorem and its generalizations ${ }^{10}$. In classical mechanics, the situation is similar: both symplectic and antisymplectic transformations are considered symmetries. So, this approach saves Curie's principle only by using standard language in a non-standard way.

4.2. Argue for a non-standard notion of invariance. A clever response is to notice that, although Earman's discussion does not mention antilinear operators, the above two propositions actually do hold when $\theta$ is antilinear. (Their proofs go through in the very same way.) However, when $\theta=T$ is the time reversal operator, the premise corresponding to (CP2) that $T e^{-i t H} T^{-1}=e^{-i t H}$ (or $\theta \alpha \theta^{-1}=\alpha$ in Earman's language) does not capture the usual notion of "invariance under time reversal." As we saw in Section 3.1. time reversal invariance is equivalent to the statement that $T H T^{-1}=H$, and since $T$ is antiunitary, this is equivalent to $T e^{-i t H} T^{-1}=e^{i t H}$. So, time reversal invariance does not mean that the dynamics is unchanged, but that it is reversed in time.

But suppose we modify our notion of invariance. One might interpret "invariance" to mean that $e^{-i t H}$ is preserved, and adopt this principle instead of Earman's premise (CP2). Then we would have,

$\left(\mathrm{CP}^{\prime}\right)$ The laws of motion/field equations governing the system are such that if an initial state is invariant under a symmetry,

\footnotetext{
${ }^{9}$ Earman states the former; the latter is similar: $\theta \psi_{1}=\theta e^{-i t H} \psi_{0}=e^{-i t H} \theta \psi_{0}=$ $e^{-i t H} \psi_{0}=\psi_{1}$.

${ }^{10}$ Cf. (Wigner 1931, §20), (Uhlhorn 1963), (Varadarajan 2007, Theorem 4.29); the latter two take a symmetry to be an automorphism of the lattice of projections, which extend to both automorphisms and the anti-automorphisms of the $C^{*}$ algebra.
} 
then so is every final state; in particular, in quantum theory, $S e^{-i t H} S^{-1}=e^{-i t H}$.

This statement, together with Earmans (CP3) "the initial state of the system is invariant under said symmetry transformation," obviously implies (CP4): "the final state of the sys- tem is also invariant under said symmetry transformation" by simple modus ponens. In particular we can verify in quantum theory that if $S \psi=\psi$ and $S e^{-i t H} S^{-1}=$ $e^{-i t H}$, then $S \psi(t)=\psi(t)$. So we have another true statement that resembles Curie's principle.

Earman may have had this condition in mind, in suggesting the equivalent statement that we "understand the invariance of laws of motion/field equations to mean that if an initial state is evolved for any chosen $\Delta t$ to produce a final state and then the symmetry operation is applied to the final state, the resulting state is the same as obtained by first applying the symmetry operation to the initial state and then evolving the resulting state for the same $\Delta t$ " (Earman 2004, p.176). On this reading of "invariance," Earman's Proposition 2 provides a correct statement, which resembles Curie's principle, and which applies to both linear and antilinear operators.

The price of this response is that one must give up the standard meaning of "time reversal invariance," in favor of a property that is almost never satisfied. The non-standard interpretation of time reversal invariance holds whenever $T e^{-i t H} T^{-1}=e^{-i t H}$. But the standard interpretation of time reversal invariance is that $T e^{-i t H} T^{-1}=e^{i t H}$. It is easy to verify ${ }^{11}$ that a system can only be simultaneously time reversal invariant in both the standard and the non-standard senses if the dynamics is trivial, $e^{-i t H}=I$. So, since almost all known quantum systems satisfy the standard definition of time reversal invariance, it follows that almost none of them satisfy the non-standard definition. In other words, the price of this approach is really to render Curie's principle inapplicable to almost every quantum system.

4.3. Argue that Curie's principle is about trajectories. A third response is to retain the orthodox definitions of symmetry and invariance but to modify the kind of object that Curie's principle is about. The last premise and the conclusion of Curie's principle (Earman's CP3 and $\mathrm{CP} 4)$ are about states. They read:

(CP3) the initial state of the system is invariant under the symmetry; (CP4) the final state of the system is invariant under said symmetry.

\footnotetext{
${ }^{11}$ If $T e^{-i t H} T^{-1}=e^{-i t H}$ and $T e^{-i t H} T^{-1}=e^{i t H}$, then $e^{-i t H}=e^{i t H}$. But Stone's theorem implies that this group has a unique generator, so $H=-H$. This is only possible if $H=0$, and hence $e^{-i t H}=I$.
} 
But premise (CP2) is about invariance of the laws, which on the standard interpretation refers to an entire trajectory. In particular (as discussed in Section 3.1), the laws are invariant under a transformation if whenever $\psi(t)$ is a possible trajectory, then so is the transformed trajectory $\psi^{\prime}\left(t^{\prime}\right)$. So, we can view the trouble with Curie's principle as one of discord between two objects interest: states in one premise, and trajectories in another.

One can bring these objects of interest into closer agreement by making all the premises of Curie's principle about trajectories. To do this, let us write $\left\{\psi(t)=e^{-i t H} \psi: t \in \mathbb{R}\right\}$ to denote the trajectory with initial state $\psi$. We begin by distinguishing two senses in which a state $\psi(t)$ in that trajectory can be "symmetric" with respect to a symmetry transformation.

(1) A state $\psi(t)$ at a time $t$ is $S$-symmetric in the original order if $S \psi(t)=\psi(t)$.

(2) A state $\psi(t)$ at a time $t$ is $S$-symmetric in the reverse order if $S \psi(t)=\psi(-t)$.

This is not such an unusual distinction, when one recalls (from the end of Section 4.1) that the standard definition of time reversal invariance entails a similar reversal of sign: $T e^{-i t H} T^{-1}=e^{i t H}$.

We can now express a revision of Curie's principle: If,

(CP1) the laws of motion/field equations governing the system are deterministic;

(CP2) the laws of motion/field equations governing the system are invariant under a symmetry transformation; and

$\left(\mathrm{CP} 3^{\prime}\right)$ the state of the system at some fixed time $t_{0}$ is symmetric under said symmetry (in the original or reverse order);

then,

$\left(\mathrm{CP} 4^{\prime}\right)$ the state of the system at any time $t$ is symmetric under said symmetry (in the same order).

In the context of ordinary quantum mechanics, this statement corresponds to the following two fact: ${ }^{12}$.

Fact 1. Suppose a state $\psi\left(t_{0}\right):=e^{-i t_{0} H} \psi$ at a fixed time $t_{0}$ is $\theta$ symmetric in the original order (i.e. $\theta \psi\left(t_{0}\right)=\psi\left(t_{0}\right)$ ), and that the unitary group $e^{-i t H}$ generating the dynamics is invariant under $\theta$ in the original order (i.e. $\theta e^{-i t H} \theta^{-1}=e^{-i t H}$ ). Then for all times $t$, the state $\psi(t)=e^{-i t H} \psi$ is $\theta$-symmetric in the same order.

\footnotetext{
${ }^{12}$ Fact 1 follows from the non-relativistic version of Proposition 2 in the last subsection. Fact 2 is proved: $T \psi(t)=T e^{-i\left(t-t_{0}\right) H} e^{-i t_{0} H} \psi=T e^{-i\left(t-t_{0}\right) H} \psi\left(t_{0}\right)=$ $e^{i\left(t-t_{0}\right) H} T \psi\left(t_{0}\right)=e^{i\left(t-t_{0}\right)} \psi\left(-t_{0}\right)=e^{i\left(t-t_{0}\right) H} e^{i t_{0} H} \psi=e^{i t H} \psi=\psi(-t)$.
} 
Fact 2. Suppose a state $\psi\left(t_{0}\right):=e^{-i t_{0} H} \psi$ at a fixed time $t_{0}$ is $\theta$ symmetric in the reverse order (i.e. $\theta \psi\left(t_{0}\right)=\psi\left(-t_{0}\right)$ ), and that the unitary group $e^{-i t H}$ generating the dynamics is invariant under $\theta$ in the reverse order (i.e. $\theta e^{-i t H} \theta^{-1}=e^{i t H}$ ). Then for all times $t$, the state $\psi(t)=e^{-i t H} \psi$ is $\theta$-symmetric in the reverse order.

We have again arrived at a correct mathematical statement. Time reversal is no longer excluded, being captured now by Fact 2. We have moreover retained the usual definition of a "symmetry/invariance" of the laws. But is this Curie's principle? Strictly speaking, Curie's principle says that if the initial state is preserved by a symmetry transformation, then so is the final state. This is not what is described by Fact 2 above, where the symmetry transformation "flips" each state about the temporal origin. Facts 1 and 2 perhaps express a more natural principle, in bringing the premises into closer alignment. But they do not capture the original expression of Curie's principle.

\section{Conclusion}

If one reads "symmetry" in the statement of Curie's principle to include time reversal symmetry, then Curie's principle is false. In particular, when we try to apply it to time reversal, Curie's principle fails for systems as elementary as the harmonic oscillator. It fails in the context of Hamiltonian mechanics, and it fails in the context of quantum theory.

There remain at least three statements in the neighborhood of Curie's principle that are mathematically correct. They can be achieved either by excluding symmetry transformations like time reversal, or by modifying the statements (CP2)-(CP4) appearing in the principle. But although these modifications may be of independent interest, I do not see that any one provides a plausible way to interpret Curie's principle. The correct conclusion, I submit, is that Curie's principle simply fails for time reversal symmetry.

\section{REFERENCES}

Brading, K. and Castellani, E. (2003). Symmetries in physics: philosophical reflections, Cambridge: Cambridge University Press.

Curie, P. (1894). Sur la symétrie dans les phénomènes physique, symétrie d'un champ électrique et d'un champ magnétique, Journal de Physique Théorique et Appliquée 3: 393-415.

Earman, J. (2002). What time reversal is and why it matters, International Studies in the Philosophy of Science 16(3): 245-264. 
Earman, J. (2004). Curie's Principle and spontaneous symmetry breaking, International Studies in the Philosophy of Science 18(2-3): 173198.

Ismael, J. (1997). Curie's Principle, Synthese 110(2): 167-190.

Messiah, A. (1999). Quantum Mechanics, Two Volumes Bound as One, New York: Dover.

Mittelstaedt, P. and Weingartner, P. A. (2005). Laws of Nature, Springer-Verlag Berlin Heidelberg.

Sachs, R. G. (1987). The Physics of Time Reversal, Chicago: University of Chicago Press.

Uhlhorn, U. (1963). Representation of symmetry transformations in quantum mechanics, Arkiv för Fysik 23: 307-340.

Varadarajan, V. S. (2007). Geometry of Quantum Theory, New York: Springer Science and Business Media, LLC.

Weinberg, S. (1995). The Quantum Theory of Fields, Vol. 1: Foundations, Cambridge: Cambridge University Press.

Wigner, E. P. (1931). Group Theory and its Application to the Quantum Mechanics of Atomic Spectra, New York: Academic Press (1959). 